\title{
CONSERVAÇÃO DE Dimorphandra mollis BENTH. (FABACEAE) BASEADA NA ESTRUTURA GENÉTICA DE POPULAÇÕES NATURAIS ${ }^{1}$
}

\author{
Ana Cecília Gonçalves ${ }^{2}$, Fábio de Almeida Vieira ${ }^{3}$, Cristiane Aparecida Fioravante Reis ${ }^{4}$ e \\ Dulcinéia de Carvalho ${ }^{4}$
}

\begin{abstract}
RESUMO - Dimorphandra mollis é uma espécie nativa do Cerrado com grande potencial econômico e tem sido alvo de intensa exploração, principalmente de seus frutos por causa do princípio ativo do composto rutina, importante para a produção de fármacos. Algumas propostas têm surgido para uma coleta controlada desses frutos, de forma a minimizar a perda de diversidade genética, entretanto existem poucas informações sobre aspectos ecológicos e genéticos da espécie. Nesse sentido, realizou-se o estudo da estrutura genética por meio de marcadores aloenzimáticos, visando dar subsídios a propostas de conservação de populações naturais de D. mollis. Dez locos polimórficos foram utilizados para estimar as frequências alélicas referentes a 180 indivíduos, distribuídos em três populações naturais (Campina Verde, Vargem da Cruz e Pau de Fruta) no Município de Jequitaí, Norte de Minas Gerais, Brasil. Os resultados indicam alta diversidade genética da espécie $\left(\hat{H}_{\bar{e}}=0,463\right)$, sendo pequena a variabilidade genética entre populações $\left(\hat{\theta_{p}}=0,025\right)$. Foi verificada ausência de endogamia dentro das populações $(\hat{f}=-0,018)$ e no conjunto das populações $(\hat{F}=0,007)$. O fluxo gênico estimado no conjunto das populações foi alto, com $\hat{N m}$ igual a 4,0, e suficiente para contrapor os efeitos da deriva genética. A alta diversidade genética nas populações da espécie indica potencial para a conservação genética in situ e também para o seu manejo. As estratégias de manejo da espécie devem considerar o tamanho efetivo populacional, no intuito de manter os níveis de variabilidade genética observados e a regeneração natural nas áreas.
\end{abstract}

Palavras-chave: Fava d’anta, Marcadores aloenzimáticos e Variabilidade genética.

\section{CONSERVATION OF Dimorphandra mollis Benth. (FABACEAE) BASED ON THE GENETIC STRUCTURE OF NATURAL POPULATIONS}

\begin{abstract}
Dimorphandra mollis is a native species of Cerrado and has high economic value due to the active compound Rutin present in its fruit, which has made it a target of intense commercial exploitation. Some proposals to minimize the negatives genetic/physiological effects of exploitation have been proposed, but little is known about the ecology or genetic structure of natural populations of this species. To delineate conservation programs, it is necessary to elucidate the levels of genetic variation in local natural populations. The genetic variability was assessed using ten allozyme loci. The genetic structure of the species was carried out using a sample of 180 individuals distributed in three natural populations located in the North of Minas Gerais, Brazil. The results showed a high level of genetic diversity within the species $\left(\hat{H}_{e}=0.463\right)$. Analysis of genetic structure indicated that most of the genetic variability of $\boldsymbol{D}$. mollis is within its natural populations with low difference among populations $\left(\hat{\theta}_{p}=0.025\right)$. Inbreeding within the populations $(\hat{f}=-0.018)$ and among them $(\hat{F}=0.007)$ was insignificant. Gene flow among populations was $\hat{N} m=4.0$, indicating to be enough to prevent the effects of genetic drift. The high genetic diversity index in these populations indicates potential for in situ genetic conservation and management. Management strategies for this species should take into consideration the effective population size in order to keep the high levels of genetic variability observed and allow natural regeneration in the areas.
\end{abstract}

Keywords: Fava d'anta, Allozyme markers and Genetic variability.

\footnotetext{
${ }^{1}$ Recebido em 20.02.2008 e aceito para publicação em 14.10.2009.

${ }^{2}$ Universidade Federal de São Carlos - Araras, SP - Brasil. E-mail: <anacecilianativa@ gmail.com>.

${ }^{3}$ Universidade Federal do Rio Grande do Norte, Unidade Acadêmica Especializada em Ciências Agrárias - Macaíba, RN Brasil. E-mail: <vieirafa@ufrnet.br>.

${ }^{4}$ Universidade Federal de Lavras - Lavras, MG - Brasil. E-mail: <cristianemep@ yahoo.com.br> e <dulce@ufla.br>.
} 


\section{INTRODUÇÃO}

No Brasil, as extensões do Cerrado chegaram a ocupar uma área heterogênea descontínua de aproximadamente 2 milhões de $\mathrm{km}^{2}$ em mais de 10 estados, representando cerca de $23 \%$ da área do território brasileiro. Nessa vasta e heterogênea região, encontrase grande variedade de sistemas ecológicos, inclusive manchas de floresta, variados tipos de solos, clima, relevo e altitude, prevalecendo, em quase toda a sua extensão, uma combinação peculiar de condições edáficas e climáticas, que deu origem à vegetação que a caracteriza (GOMES, 1998). É considerada uma das áreas mais ricas do mundo em diversidade biológica (MYERS et al., 2000; MITTERMEIER et al., 2000), com alto endemismo. A partir da década de 1950, vários de seus ecossistemas deram lugar à pecuária e à agricultura extensiva, em detrimento de uma diversidade biológica até então pouco alterada e desconhecida. Atualmente mais de $70 \%$ das áreas do Cerrado são "altamente modificadas", devido aos desmatamentos, queimadas, uso de fertilizantes químicos e agrotóxicos, restando menos de $20 \%$ das áreas em estado conservado, consideradas críticas para a conservação, sendo classificadas como hotspots (MITTERMEIER et al., 2000).

Dimorphandra mollis Benth. (Fabaceae) possui ampla distribuição no Cerrado. Conhecida popularmente como faveira, favela e fava d'anta, ocorre principalmente nos Estados de Mato Grosso, Goiás, Minas Gerais e São Paulo. Tipicamente de hábito xerofítico, ocorre nas variações do Cerrado (campo cerrado e cerradão) e nas matas de encosta, próximo às elevações, sendo encontrada em solos argilosos ou arenosos e em altitudes que variam entre $500 \mathrm{~m}$ e $1.700 \mathrm{~m}$. A espécie é hermafrodita, sendo as flores polinizadas por pequenos insetos. A dispersão dos propágulos é feita por mamíferos não voadores (mastocoria). Bizerril et al. (2005) analisaram amostras de fezes de anta (Tapirus terrestris) no Cerrado do Brasil Central e apontaram que este é um importante consumidor de frutos e potencial dispersor das sementes da D. mollis. Contudo, o consumo dos frutos pode ser reduzido em função da baixa densidade desses frugívoros; além disso, há a possibilidade de os dispersores originais pertencerem à megafauna extinta de mamíferos da América do Sul (BIZERRIL et al., 2005).

A principal importância econômica da espécie é o fruto (favas), pois o pericarpo e a polpa contêm glicosídeos flavônicos, especialmente a rutina (quercetina-3-rutinosídio), amplamente explorada pela indústria farmacêutica e cosmética. A rutina desponta como uma das substâncias mais promissoras na produção de medicamentos que atuam no combate ao envelhecimento e às doenças degenerativas, principalmente considerando o contínuo aumento da população idosa. No entanto, a espécie não é cultivada, e toda essa matéria-prima é extraída do Cerrado de forma desordenada, não havendo o cuidado com a reprodução da espécie (ALMEIDA LIMA, 1997). Com isso, as populações naturais de $D$. mollis podem ser extintas localmente, pois a falta de manejo pode conduzir à redução no número de indivíduos em função da ausência de critérios para a colheita das favas (GOMES, 1998). Deve-se considerar ainda que a coleta dos frutos sem um plano de conservação genética pode resultar no estreitamento da variabilidade genética, devido às restrições na disseminação de sementes e, consequentemente, com reflexos no sucesso reprodutivo e na regeneração natural da espécie.

Assim, o conhecimento da estrutura e da diversidade genética das populações naturais de $D$. mollis, bem como a compreensão de outros fatores relacionados, é um ponto- chave para a adoção de estratégias de manejo adequadas para a conservação genética da espécie. Especificamente, este trabalho teve como objetivos estudar a distribuição da variabilidade genética entre e dentro das populações, estimar o fluxo gênico e o tamanho efetivo populacional e gerar informações para a conservação genética in situ das populações naturais da espécie.

\section{MATERIAL E MÉTODOS}

\subsection{Locais de estudo e amostragem}

Foram estudadas três populações naturais de D. mollis, localizadas no Município de Jequitaí, Norte do Estado de Minas Gerais, com distâncias e altitudes variáveis. As populações estão localizadas em áreas particulares com diferentes estádios de conservação e histórico de exploração da espécie. A população Vargem da Cruz encontra-se às margens do rio Jequitaí, afluente do rio São Francisco, nas coordenadas $17^{\circ} 10^{\prime} 30^{\prime}$ 'S e 4429'55'W e altitude de $541 \mathrm{~m}$. Segundo a descrição de antigos moradores e catadores de fava, a área era coberta por vegetação de Cerrado sensu stricto (IBGE, 1993) e correspondia a uma área de extrativismo da espécie. Atualmente, a área apresentava- 
se altamente antropizada, e as árvores ocorriam em área de pastagens. Nesse local, havia ainda árvores remanescentes da espécie em razão do grande interesse na coleta dos frutos, que é realizada pelo proprietário, moradores meeiros e outros catadores da região. A população Campina Verde, nas coordenadas $17^{\circ} 12^{\prime} 34^{\prime \prime} S$ e 44⒉'20"W e altitude de $530 \mathrm{~m}$, apresenta vegetação explorada, principalmente, para a produção do carvão vegetal. Os moradores dessa área, assim como aqueles de Vargem da Cruz, fazem o corte "seletivo", deixando indivíduos de D. mollis para a coleta das favas. Entretanto, percebem-se no campo várias árvores da espécie cortada. A população Pau de Fruta, nas coordenadas $17^{\circ} 13^{\prime} 20^{\prime \prime}$ S e 4421'46"W, estava localizada em uma chapada de difícil acesso e de maior altitude $(760 \mathrm{~m})$. Era uma região divisora de águas, que permanecia com vegetação remanescente de Cerrado, na formação original sensu stricto, ainda pouco explorada, o que permitia que as árvores da espécie se mantinham mais protegidas do extrativismo local. A distância entre as populações Campina Verde e Vargem da Cruz era de 11,33 km, entre Campina Verde e Pau de Fruta 5,42 km e entre Vargem da Cruz e Pau de Fruta de 16,20 km.

Em cada uma das populações foram amostradas, ao acaso, 60 árvores adultas com uma distância mínima de $50 \mathrm{~m}$ entre cada uma. Todas as árvores foram plaqueteadas no caule com número correspondente e georreferenciadas com aparelho GPS. Amostras foliares foram coletadas e colocadas em sacos plásticos identificados e acondicionados em caixa térmica com gelo e transportadas até o laboratório, onde foram armazenadas a $-75^{\circ} \mathrm{C}$. A extração das enzimas foi realizada no Laboratório de Conservação Genética de Espécies Arbóreas do Departamento de Ciências Florestais/UFLA.

\subsection{Procedimentos para a eletroforese e revelação dos géis}

No procedimento de extração, foram utilizados 350 $\mathrm{mg}$ de tecido foliar, polivinil-pirrolidona-PVP e 1,75 $\mathrm{mL}$ da solução-tampão $\mathrm{n}^{\circ} 1$ descrita em Alfenas et al. (1998). As amostras foliares de cada árvore foram maceradas manualmente com o uso de almofariz e pistilos de porcelana mantidos resfriados durante o processo. A eletroforese foi conduzida em cuba vertical, utilizando gel de poliacrilamida como suporte, sendo $4,5 \%$ o gel de concentração e 12,5\% o gel de separação. Foi utilizada amperagem de $10 \mathrm{~mA}$ por gel, 300 Volts, tendo a corrida eletroforética duração de 3,5 h. Foram testados 15 sistemas enzimáticos (ALFENAS et al., 1998), sendo selecionados os que apresentaram locos e alelos passíveis de interpretação: álcool desidrogenase (ADH), b-Galactose desidrogenase (GLDH), fosfatase ácida (ACP), glucose desidrogenase $(\mathrm{GDH})$, glutamato desidrogenase (GTDH), malato desidrogenase (MDH), peroxidase $(\mathrm{PO})$, sorbitol desidrogenase $(\mathrm{SDH})$ e xiquimato desidrogenase (SKDH). A identificação dos locos e dos alelos foi feita a partir da região mais catódica para a mais anódica.

\subsection{Análise dos dados}

A variabilidade genética de cada população foi caracterizada pela estimativa das frequências alélicas e dos índices de diversidade: número médio de alelos por loco $(\hat{A})$; porcentagem de locos polimórficos $\left(P_{L}\right.$; critério 0,95$)$; heterozigosidades esperada $(\hat{H})$ e observada $\left(\hat{H}_{o}\right)$ e a estimativa do coeficiente de endogamia $(\hat{f})$ (MORAES et al., 2005). Para obtenção dessas estimativas, foi utilizado o software BIOSYS 2 (SWOFFORD e SELANDER, 1997). Foram considerados locos em equidade gênica (baixa amplitude de variação das frequências alélicas) quando eles apresentavam frequências alélicas entre 0,35 e 0,65 (FRANKEL et al., 1995).

Foram estimados os seguintes coeficientes de coancestralidade: $\hat{f}$, que é a média do desvio de panmixia (endogamia devida ao cruzamento entre parentes) das populações; $\hat{\theta_{p}}$, a endogamia (perda de heterozigosidade) devida à subdivisão da população, que reflete a diversidade das populações; $\hat{F}$ e, que é a endogamia devida às duas causas. Para verificar se essas estimativas médias eram diferentes de zero, estimou-se o intervalo de confiança com $95 \%$ de probabilidade, pelo método de reamostragem bootstrap, utilizando-se 10.000 repetições sobre locos, com o auxílio do programa GDA (LEWIS e ZAYKIN, 2000). As estimativas do fluxo gênico $(\hat{N} m)$ entre as populações foram obtidas segundo a equação proposta por Crow e Aoki (1984): $\hat{N} m=1 /$ 4á $\left(1 / \hat{\theta_{p}}-1\right)$, em que $\alpha=(n /(n-1))^{2}$, sendo $n$ o número de populações. O tamanho efetivo da população foi estimado pela razão $\hat{N}_{e} / n$, em que $\hat{N}_{e}=n / 1+\hat{f}$, sendo $n$ o número de plantas amostradas e $\hat{f}$ o coeficiente de endogamia intrapopulacional (VENCOVSKY, 1997). Valores que se desviam da unidade significam que na amostragem das populações existe deficiência de indivíduos para contribuir com cruzamentos aleatórios.

R. Árvore, Viçosa-MG, v.34, n.1, p.95-101, 2010 


\section{RESULTADOS}

Foram obtidas as frequências de 20 alelos distribuídos em 10 locos aloenzimáticos das três populações naturais de D. mollis (Tabela 1). A população Campina Verde apresentou o maior número de alelos com alta frequência, ACP-1 (alelo 2), ACP-2 (alelo 1), GLDH (alelo 2) e MDH (alelo 2); a população Vargem da Cruz exibiu três alelos com alta frequência, ACP-1 (alelo 1), ACP-2 (alelo 1) e ADH (alelo 2). A população Pau de Fruta mostrou apenas um alelo com alta frequência ACP-2 (alelo 1). Considerando que locos em equidade gênica apresentam frequências alélicas entre 0,35 e 0,65, observou-se, na população Campina Verde, $60 \%$ dos locos em equidade gênica, $70 \%$ na população Vargem da Cruz e de $90 \%$ na população Pau de Fruta.

Tabela 1 - Frequências alélicas e tamanho da amostra $(n)$ em 10 locos aloenzimáticos, em populações naturais de Dimorphandra mollis.

Table 1 - Allele frequencies and sample size (n), in 10 allozyme loci, of natural populations of Dimorphandra mollis.

\begin{tabular}{|c|c|c|c|c|}
\hline \multicolumn{5}{|c|}{ Populações } \\
\hline Loco & Alelo & $\begin{array}{l}\text { Campina } \\
\text { Verde }\end{array}$ & $\begin{array}{l}\text { Vargem } \\
\text { da Cruz }\end{array}$ & $\begin{array}{c}\text { Pau de } \\
\text { Fruta }\end{array}$ \\
\hline \multirow[t]{3}{*}{$\overline{\mathrm{ACP}-1}$} & 1 & 0,322 & 0,750 & 0,385 \\
\hline & 2 & 0,678 & 0,250 & 0,615 \\
\hline & $n$ & 45 & 42 & 48 \\
\hline \multirow[t]{3}{*}{$\mathrm{ACP}-2$} & 1 & 0,744 & 0,774 & 0,781 \\
\hline & 2 & 0,256 & 0,226 & 0,219 \\
\hline & $n$ & 45 & 42 & 48 \\
\hline \multirow[t]{3}{*}{$\mathrm{ADH}$} & 1 & 0,359 & 0,294 & 0,354 \\
\hline & 2 & 0,641 & 0,706 & 0,646 \\
\hline & $n$ & 46 & 51 & 48 \\
\hline \multirow[t]{3}{*}{ GDH } & 1 & 0,412 & 0,458 & 0,400 \\
\hline & 2 & 0,588 & 0,542 & 0,600 \\
\hline & $n$ & 51 & 59 & 50 \\
\hline \multirow{3}{*}{ GLDH } & 1 & 0,255 & 0,387 & 0,408 \\
\hline & 2 & 0,745 & 0,613 & 0,592 \\
\hline & $n$ & 47 & 53 & 49 \\
\hline \multirow[t]{3}{*}{ GTDH } & 1 & 0,419 & 0,417 & 0,448 \\
\hline & 2 & 0,581 & 0,583 & 0,552 \\
\hline & $n$ & 43 & 60 & 48 \\
\hline \multirow[t]{3}{*}{$\mathrm{MDH}$} & 1 & 0,340 & 0,474 & 0,451 \\
\hline & 2 & 0,660 & 0,526 & 0,549 \\
\hline & $n$ & 50 & 57 & 51 \\
\hline \multirow[t]{3}{*}{$\mathrm{PO}$} & 1 & 0,472 & 0,602 & 0,420 \\
\hline & 2 & 0,528 & 0,398 & 0,580 \\
\hline & $n$ & 53 & 59 & 56 \\
\hline \multirow[t]{3}{*}{ SDH } & 1 & 0,394 & 0,407 & 0,372 \\
\hline & 2 & 0,606 & 0,593 & 0,628 \\
\hline & $n$ & 47 & 59 & 43 \\
\hline \multirow[t]{3}{*}{ SKDH } & 1 & 0,561 & 0,500 & 0,373 \\
\hline & 2 & 0,439 & 0,500 & 0,627 \\
\hline & $n$ & 49 & 58 & 51 \\
\hline
\end{tabular}

Tabela 2-Índices de diversidade genética em populações de Dimorphandra mollis. Â, número médio de alelos por loco gênico; $P_{0.05}$, porcentagem dos locos polimórficos; $\hat{H}_{o}$, heterozigosidade média observada; $\hat{H}_{e}$, heterozigosidade média esperada; $\hat{f}$ índice de fixação de Wright; e $\hat{N}_{e}$, tamanho efetivo populacional.

Table 2 - Genetic diversity index in populations of Dimorphandra mollis. Â, average number of alleles per locus; $P_{0.05}$, proportion of polymorphic loci; $\hat{H}_{o}$, observed heterozygosity; $\hat{H}_{\mathfrak{e}}$, expected heterozygosity; $\hat{f}$, fixation index; $\hat{N}_{e}$, effective size

\begin{tabular}{lccc}
\hline & \multicolumn{4}{c}{ Populações } \\
\hline & $\begin{array}{c}\text { Campina } \\
\text { Verde }\end{array}$ & $\begin{array}{c}\text { Vargem } \\
\text { da Cruz }\end{array}$ & $\begin{array}{c}\text { Pau } \\
\text { de Fruta }\end{array}$ \\
\hline$\hat{A}$ & 2,0 & 2,0 & 2,0 \\
$P_{0,05}$ & 100,0 & 100,0 & 100,0 \\
$\hat{H}_{o}$ & $0,449(0,071)$ & $0,494(0,058)$ & $0,462(0,018)$ \\
$\hat{H}_{e}$ & $0,459(0,014)$ & $0,460(0,017)$ & $0,470(0,014)$ \\
$\hat{f}$ & 0,023 ns & $-0,075$ ns & 0,017 ns \\
& {$[-0,253$ a 0,267$]$} & {$[-0,262$ a 0,134$]$} & {$[-0,074$ a 0,109$]$} \\
$n$ & 60 & 60 & 60 \\
$\hat{N}_{e} / n$ & 0,98 & 1,00 & 0,98 \\
\hline
\end{tabular}

. $n$, tamanho amostral; ( ), erro-padrão; [ ], intervalo de confiança $(\alpha=0,05)$; e ns, não significativo.

Tabela 3 -Divergência genética $\left(\hat{\theta}_{p}\right)$ e fluxo gênico $\hat{N} m$ entre populações de Dimorphandra mollis.

Table 3 - Genetic divergence $\left(\hat{\theta}_{p}\right)$ and gene flow $\hat{N} m$ between populations of Dimorphandra mollis.

\begin{tabular}{cccc}
\hline Populações & \multicolumn{3}{c}{ Distância } \\
\hline $\begin{array}{c}\text { Campina Verde } \\
\text { e Vargem da } \\
\text { Cruz }\end{array}$ & $11,33 \mathrm{~km}$ & $0,042[-0,002$ a 0,112$]$ & 1,4 \\
$\begin{array}{c}\text { Campina Verde } \\
\text { e Pau de Fruta }\end{array}$ & $5,42 \mathrm{~km}$ & $0,007[-0,007$ a 0,022$]$ & 8,9 \\
$\begin{array}{c}\text { Vargem da Cruz } \\
\text { e Pau de Fruta } \\
\begin{array}{c}\text { Todas as } \\
\text { populações }\end{array}\end{array}$ & $16,20 \mathrm{~km}$ & $0,031[-0,004$ a 0,083$]$ & 1,9 \\
\hline
\end{tabular}

[ ] = intervalo de confiança $(\alpha=0,05)$.

O percentual de locos polimórficos $\left(P_{0,05}\right)$, utilizando como critério uma frequência de $95 \%$ para o alelo mais comum, foi de $100 \%$ nos locos detectados (Tabela 2). O número de alelos por loco $(\hat{A})$ foi de 2,0 em cada uma das populações. A heterozigosidade média esperada ( $\hat{H}_{e}$ ) nas populações de $D$. mollis variou de 0,459 a 0,470 e a observada $\left(\hat{H}_{o}\right)$, de 0,449 a 0,494 . A relação entre as heterozigosidades médias observadas e as esperadas forneceu o índice de fixação $(\hat{f})$ não significativo das populações. 
As estimativas médias obtidas dos coeficientes de coancestralidade de Cockerham indicaram ausência de endogamia dentro das populações $[\hat{f}=-0,018$ $(-0,126$ a 0,090$)]$ e no conjunto das populações $[\hat{F}=0,007(-0,118$ a 0,139$)]$, conforme intervalo de confiança. A divergência genética entre populações foi baixa $\left[\hat{\theta}_{p}=0,025(-0,004\right.$ a 0,065$\left.)\right]$. Isso significa que aproximadamente $2,5 \%$ da variabilidade genética encontra-se entre as populações e que $97,5 \%$ dessa variabilidade ocorre dentro das populações (Tabela 3).

As estimativas de fluxo gênico histórico $(\hat{\mathrm{N} m})$ encontradas no conjunto das populações foram de quatro migrantes por geração, indicando considerável fluxo gênico aparente (Tabela 3). A razão entre o tamanho efetivo das populações e o número de plantas amostradas foi próxima a 1,0 (Tabela 3).

\section{DISCUSSÃO}

Na análise das frequências alélicas foram observados alelos com maior frequência e menor porcentagem de locos em equidade gênica nas populações onde a espécie é explorada desordenadamente. A equidade gênica é um indicador da diversidade genética das populações. Dessa forma, populações que apresentam maior variação das frequências alélicas (menor equidade gênica) estão teoricamente mais suscetíveis à fixação e perda de alelos quando submetidas a perturbações e gargalos genéticos (FRANKEL et al., 1995). Considerando que se houver a continuidade do extrativismo intensivo dos frutos das árvores, a variabilidade genética das populações Campina Verde e Vargem da Cruz poderá ser reduzida nas próximas gerações.

O percentual de locos polimórficos e o número de alelos por loco nas populações de $D$. mollis são próximos aos encontrados para várias espécies arbóreas em estudos utilizando marcadores aloenzimáticos (BOTREL e CARVALHO, 2004; MORAES et al., 2005; GUSSON et al., 2006; VIEIRA e CARVALHO, 2008). A ausência de alelos raros e as frequências alélicas distribuídas uniformemente, por certo, contribuíram para a alta diversidade genética, com heterozigosidade total média $\left(\hat{H}_{e}\right)$ de 0,463 . Esse valor é próximo ao relatado em populações de outras espécies arbóreas de Cerrado, mesmo exploradas pelo extrativismo tradicional dos frutos, como para Annona crassiflora ( $\hat{H}_{e}=0,357$; TELLES et al., 2003) e Caryocar brasiliense ( $\hat{H}_{e}^{e}=0,499$; MELO JÚNIOR et al., 2004). Em condições naturais de reprodução sexual, a alta diversidade encontrada em $D$. mollis indicaria a possibilidade da ocorrência de numerosas novas combinações genotípicas, aumentando o potencial evolutivo e a capacidade de adaptação da espécie às possíveis mudanças ambientais. De fato, os índices de fixação $(\hat{f})$ indicaram que as populações são panmíticas, não se desviando do Equilíbrio de Hardy Weinberg. Entretanto, a exploração desordenada dos frutos, impedindo a dispersão e, consequentemente, o estabelecimento natural das plântulas, poderá ocasionar a perda da diversidade genética nas próximas gerações dessas populações. Seria importante que estudos de estrutura genética fossem realizados também nas próximas gerações.

D. mollis é polinizada por insetos e apresenta frutos de dispersão zoocórica, portanto baixa diferenciação genética entre populações era esperada. Em geral, estudos com outras espécies vegetais em regiões de Cerrado mostram maior proporção da variabilidade genética dentro das populações (MELO JÚNIOR et al., 2004; FRANCESCHINELLI et al., 2006; TELLES et al., 2003). Espécies tipicamente alógamas apresentam alta variabilidade genética dentro de populações (LOVELESS e HAMRICK, 1984); a divergência genética entre populações é reduzida com o aumento do fluxo gênico (pólen e, ou, sementes).

Os valores de $\hat{N} m$ determinam se a deriva genética, por si só, pode produzir variabilidade genética substancial entre locais. Se esses valores forem superiores a 1,0, então o fluxo gênico será alto o suficiente para prevenir a diferenciação devido à deriva genética (SLATKIN e BARTON, 1989). Entre as três populações, pôde-se verificar maior fluxo gênico entre Campina Verde e Pau de Fruta, indicando maior similaridade genética provavelmente devido ao fluxo antigo de alelos. Possivelmente, essa relação se deve à proximidade geográfica entre essas duas populações, conforme modelo de isolamento pela distância. O fluxo gênico entre as populações Campina Verde e Pau de Fruta excedeu quatro migrantes por geração $(\hat{N} m=8,9)$, sugerindo populações panmíticas (HARTL e CLARK, 1997).

Altos níveis de fluxo gênico poderiam, em condições naturais, diminuir a perda de diversidade genética e manter a viabilidade das populações no longo prazo. Entretanto, considerando a situação praticamente irreversível da fragmentação das populações de $D$. mollis e o extrativismo dos frutos impedindo a dispersão natural, há possibilidades de aumento nos níveis de diferenciação genética entre essas populações nas

R. Árvore, Viçosa-MG, v.34, n.1, p.95-101, 2010 
próximas gerações. Portanto, seria interessante realizar estudos genéticos nas progênies, para avaliar o fluxo alélico contemporâneo. Adicionalmente, a dispersão dos propágulos por mastocoria pode ser reduzida em função da baixa ocorrência desses frugívoros nas regiões de Cerrado (BIZERRIL et al., 2005).

A relação entre o tamanho efetivo populacional e o tamanho da amostra $\left(\hat{N}_{e} / n\right)$ é de grande importância para o planejamento da conservação in situ, indicando a representatividade genética das amostras de plantas. A estimativa está associada com as condições de heterozigose, e, portanto, os valores observados $\left(\hat{N}_{e} / n \sim 1,0\right)$ reafirmam a existência de baixa endogamia para a espécie nas populações estudadas. Estratégias de conservação para as populações devem considerar a possibilidade da redução do tamanho efetivo populacional, em função da coleta extrativista dos frutos e do corte das árvores para a produção do carvão vegetal. Portanto, seria necessário definir, nas populações, árvores- matriz como porta-sementes para permitir a dispersão dos frutos, propagação e regeneração natural. Essa medida contribuiria para a manutenção dos níveis de variabilidade genética e o mínimo de endogamia, uma vez que grande quantidade de frutos é coletada, sem critérios que visem à conservação da espécie. Essa seria uma medida importante, considerando-se a relevância de D. mollis para as populações tradicionais.

\section{CONCLUSÕES}

- A diversidade genética intrapopulacional é alta, e as populações de $D$. mollis estudadas não são endogâmicas, mostrando-se potenciais para a conservação genética in situ e manejo florestal.

- Há baixa variabilidade nas frequências alélicas entre as populações, e a maior parte da variabilidade genética da espécie se encontra dentro das populações naturais.

- Estratégias de manejo da espécie devem considerar o tamanho efetivo populacional, no intuito de manter os níveis de variabilidade genética observados e a regeneração natural nas áreas.

\section{AGRADECIMENTOS}

Ao CNPq, pela concessão das bolsas de Mestrado e Produtividade em Pesquisa ao primeiro e ao último autor; à CAPES, pela concessão de bolsa de doutorado ao terceiro autor; e à FAPEMIG, pelo apoio financeiro. Finalmente, a todos que, de alguma forma, participaram da realização deste trabalho.

\section{REFERÊNCIAS}

ALFENAS, A. C. et al. Extração de proteínas para eletroforese. In: ALFENAS, A. C. (Ed.).

Eletroforese de isoenzimas e proteínas afins: fundamentos e aplicações em plantas e microrganismos. Viçosa, MG: Universidade Federal de Viçosa, 1998. p.85-114.

ALMEIDA LIMA, C. S. Desenvolvimento de um modelo para manejo sustentado do Cerrado. 1997. 159f. Dissertação (Mestrado em Engenharia Florestal) - Universidade Federal de Lavras, Lavras, 1997.

BIZERRIL, M. X. A.; RODRIGUES, F. H. G.; HASS, A. Fruit consumption and seed dispersal of Dimorphandra mollis Benth. (Leguminosae) by the lowland tapir in the Cerrado of Central Brazil. Brazilian Journal of Biology, v.65, n.3, p.407-413, 2005

BOTREL, M. C. G.; CARVALHO, D. Variabilidade isoenzimática em populações naturais de jacarandá paulista (Machaerium villosum Vog.). Revista Brasileira de Botânica, v.27, n.4, p.621-627, 2004.

CROW, J. F.; AOKI, K. Group selection for polygenic behavioral trait: estimating the degree of population subdivision. Proceedings of the Natural Academy of Sciences of the United States of America, v.81, n.19, p.6073-6077, 1984.

FRANCESCHINELLI, E. V. et al. The genetic diversity of two Brazilian Vellozia (Velloziaceae) with different patterns of spatial distribution and pollination biology. Annals of Botany, v.97, p.585-592, 2006.

FRANKEL, O. H.; BROWN, A. H. D.; BURDON, J. J. The conservation of plant biodiversity. Cambridge: Cambridge University Press, 1995. p.299.

GOMES, L. J. Extrativismo e comercialização da fava d'anta (Dimorphandra sp.) estudo de caso na região de cerrado de Minas Gerais. 1998. 158f. Dissertação (Mestrado em Engenharia Florestal) - Universidade Federal de Lavras, Lavras, 1998. 
GUSSON, E.; SEBBENN, A. M.; KAGEYAMA, P. Y. Sistema de reprodução em populações de Eschweilera ovata (Cambess.) Miers. Revista Árvore, v.30, n.4, p.491-502, 2006.

HARTL, D. L.; CLARK, A. G. Principles of population genetics. Sunderland: Sinauer Associates, 1997.

\section{INSTITUTO BRASILEIRO DE GEOGRAFIA E ESTATÍSTICA - IBGE. Classificação da vegetação brasileira. São Paulo: 1993. 89p.}

LEWIS, P. O.; ZAYKIN, D. Genetic data analysis: Computer program for the analysis of allelic data. Version 1.0 (d15). Free program distributed by the authors over the internet from the GDA. 2000.

LOVELESS, M. D.; HAMRICK, J. L. Ecological determinants of genetic structure in plant populations. Annual Review of Ecology and Systematics, v.15, p.65-95, 1984.

MELO JUNIOR, A. F. et al. Estrutura genética de populações naturais de pequizeiro (Caryocar brasiliense Camb.). Scientia Forestalis, v.66, p.56-65, 2004.

MITTERMEIER, R. A.; MYERS, N.; MITTERMEIER, C. G. (Eds.). Hotspots: earth s biologically richest and most endangered terrestrial ecoregions. México: CEMEX/ Conservation International, 2000. 431p.

MYERS, N. et al. Biodiversity hotspots for conservation priorities. Nature, v.403, p.853-858, 2000.
MORAES, M. L. T.; KAGEYAMA, P. Y.; SEBBENN A. M. Diversidade e estrutura genética espacial em duas populações de Myracrodruon urundeuva Fr. All. sob diferentes condições antrópicas. Revista Árvore, v.29, n.2, p.281-289, 2005.

SLATKIN, M.; BARTON, N. H. A comparison of three methods for estimating average levels of gene flow. Evolution, v.43, n.7, p.1349$1368,1989$.

SWOFFORD, D. L.; SELANDER, R. B. Biosys-2: a computer program for the analysis of allelic variation in population genetics and biochemical systematics. Urbana: University of Illinois, 1997.

TELLES, M. P. C. et al. Caracterização genética de populações naturais de araticunzeiro (Annona crassiflora Mart. - Annonaceae) no Estado de Goiás. Revista Brasileira de Botânica, v.26, n.1, p.123-129, 2003.

VENCOVSKY, R. Biometrical approaches for molecular markers: estimation of effective population size. In: INTERNATIONAL WORKSHOP ON AGRICULTURAL BIOTECHNOLOGY, 1997, Piracicaba. Proceedings... Piracicaba: ESALQ-USP; Cook College: New Jersey Agricultural Experiment Station, The State University of New Jersey, 1997. 2p.

VIEIRA, F. A.; CARVALHO, D. Genetic structure of an insect-pollinated and bird dispersed tropical tree in vegetation fragments and corridors: implications for conservation. Biodiversity and Conservation, v.17, n.10, p.2305-2321, 2008. 
